\title{
Does the evolution of viral polymerases reflect the origin and evolution of viruses?
}

\section{Mart Krupovič and Dennis H. Bamford}

A swiftly increasing amount of available genomic information allures scientists to systematize the data and embed it into evolutionary scenarios. The recent analysis of viral RNA-dependent RNA polymerase (RdRp), superfamily 3 helicase and chymotrypsinlike protease sequences by Koonin and co-workers (Big Bang of picorna-like virus evolution antedates the radiation of eukaryotic supergroups. Nature Rev. Microbiol. 6, 925-939 (2008)) $)^{1}$ clusters a number of RNA viruses into the picorna-like superfamily and led to the proposal that this group has a monophyletic origin. This superfamily, which includes viruses in the order Picornavirales, is now proposed to include other viruses from ten viral families as well as several as-yetunclassified virus species. The characteristic feature of classical icosahedral picorna-like viruses is the jelly-roll capsid protein fold ${ }^{2}$. The new proposal by Koonin et al. combines both icosahedral (single-stranded RNA (ssRNA) and double-stranded RNA (dsRNA)) and helical viruses that have no similarities in virion architecture. The common nominator for all these viruses is the presence of a gene that encodes a related RdRp.

But how do we define a virus? It is widely accepted that a replicon is a virus only when it can produce a virion. Consequently, virion architecture, as well as the structures of the

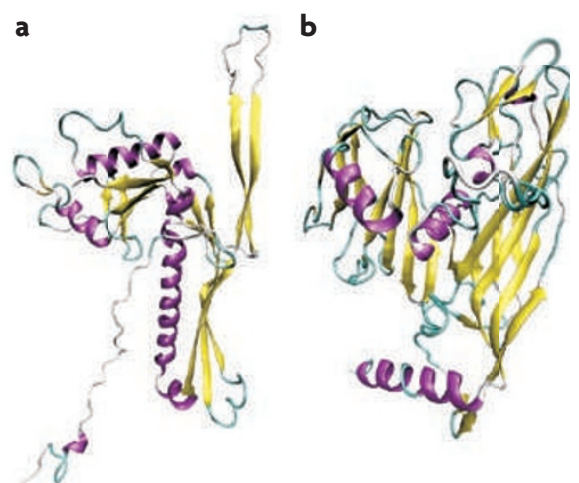

Figure 1 | Comparison of the main capsid protein X-ray structures of bacteriophages. The structures of HK97 (a; protein data bank code 1OHG) and PRD1 (b; protein data bank code 1 $\underline{1 \mathrm{HX} 6}$ ) are shown. These two viruses assemble unrelated virion structures in distinct and different ways. major components of the virion, are crucial when considering the origins and evolution of viruses ${ }^{3}$. In other words, a replicon transforms to a virus only after acquisition of genetic determinants for the virion. It follows that when we analyse relationships between viruses, and not only replicons (for example, plasmids and transposons), a virion-centred approach must dominate. It is evident that replicases can be horizontally exchanged between unrelated viruses. For example, tailed phi29-like bacteriophages (which have an HK97 capsid protein fold ${ }^{4,5}$ (FIG. 1a); Podoviridae family) and tailless membranecontaining bacteriophages of the Tectiviridae family (which have a double $\beta$-barrel capsid protein fold ${ }^{6}$ (FIG. 1 b)) use homologous protein-primed type B polymerases for genome replication ${ }^{7}$. However, these two viruses have no similarities in virion structure and assem$\mathrm{bly}^{8}$, a strictly regulated process that requires a concerted interplay between a number of different proteins. Similarly, different corticoviruses seem to use four non-homologous replication proteins ${ }^{9}$. Therefore, relationships between viral replication proteins do not necessarily reflect the relationships between viruses that use these proteins (FIG. 2). The extent of horizontal gene exchange between unrelated viruses and between viruses and their hosts ${ }^{10}$ emphasizes the choices to use a range of replication strategies that viruses have at their disposition.

We note that the jelly-roll fold is common to most of the icosahedral ssRNA eukaryotic viruses and is likely to reflect a monophyletic origin for these viruses ${ }^{2}$. However, the jelly-roll fold, as well as the virion assembly principles of picornaviruses, is unrelated to the capsid proteins of dsRNA viruses, such as Saccharomyces cerevisiae L-A virus (Totiviridae family), which in turn was found to share architectural principles with other dsRNA viruses that infect eukaryotes (Reoviridae family) and bacteria (Cystoviridae family) ${ }^{8}$.

The phylogenetic analysis presented by Koonin et al. ${ }^{1}$ reflects the relatedness of viral RdRps. However, owing to reasons presented above, the obtained trees do not necessarily represent relationships between viruses.

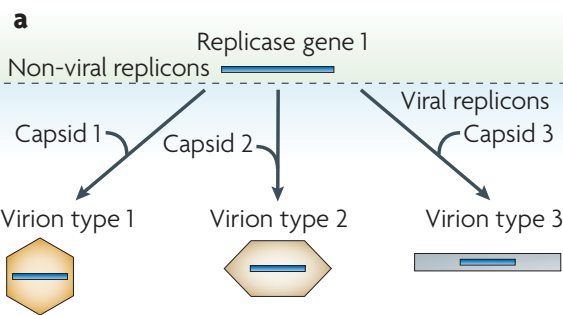

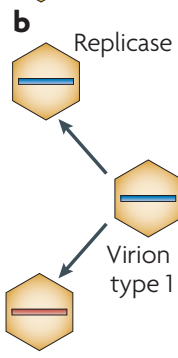

Replicase type 2

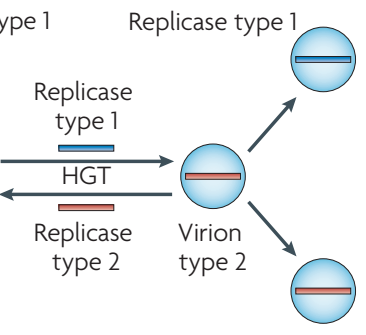

Replicase type 2
Figure 2 | Evolution of polymerases does not necessarily reflect the evolution of viruses. a Three independent instances in which virogenesis gave rise to three distinct viruses that, nevertheless, replicate their genomes with the aid of related proteins. $\mathbf{b}$ |Horizontal exchange of the replicase genes between virion types 1 and 2 does not transform virion type 1 into virion type 2 . HGT, horizontal gene transfer.

1. Koonin, E. V., Wolf, Y. I., Nagasaki, K. \& Dolja, V. V. The Big Bang of picorna-like virus evolution antedates the radiation of eukaryotic supergroups. Nature Rev. Microbiol. 6. 925-939 (2008).

2. Rossmann, M. G. \& Johnson, J. E. Icosahedral RNA virus structure. Annu. Rev. Biochem. 58, 533-573 (1989).

3. Krupovic, M. \& Bamford, D. H. Virus evolution: how far does the double $\beta$-barrel viral lineage extend? Nature Rev. Microbiol. 6, 941-948 (2008).

4. Wikoff, W. R., Liljas, L., Duda, R. L., Tsuruta, H., Hendrix, R. W. \& Johnson, J. E. Topologically linked rings of covalently joined protein subunits from the dsDNA bacteriophage HK97 capsid. Science 289. 2129-2133 (2000)

5. Morais, M. C., Choi, K. H., Koti, J. S., Chipman, P. R., Anderson, D. L. \& Rossmann, M. G. Conservation of the capsid structure in tailed dsDNA bacteriophages: the pseudoatomic structure of $\varphi 29$. Mol. Cell 18 149-159 (2005)

6. Benson, S. D., Bamford, J. K., Bamford, D. H. \& Burnett, R. M. Viral evolution revealed by bacteriophage PRD1 and human adenovirus coat protein structures. Cell 98, 825-833 (1999).

7. Rohwer, F. \& Edwards, R. The Phage Proteomic Tree: a genome-based taxonomy for phage. J. Bacteriol. 184 4529-4535 (2002).

8. Bamford, D. H., Grimes, J. M. \& Stuart, D. I. What does structure tell us about virus evolution? Curr. Opin. Struct. Biol. 15, 655-663 (2005).

9. Krupovič, M. \& Bamford, D. H. Putative prophages related to lytic tailless marine dsDNA phage PM2 are widespread in the genomes of aquatic bacteria. BMC Genomics 8, 236 (2007).

10. Hendrix, R. W., Smith, M. C., Burns, R. N., Ford, M. E. $\&$ Hatfull, G. F. Evolutionary relationships among diverse bacteriophages and prophages: all the world's a phage. Proc. Natl Acad. Sci. USA 96, 2192-2197 (1999).

Mart Krupovič and Dennis H. Bamford are at the Department of Biological and Environmental Sciences and Institute of Biotechnology, Biocenter 2, PO BOX 56 (Viikinkaari 5), 00014 University of Helsinki, Finland. Correspondence to D.H.B. e-mail: dennis.bamford@helsinki.fi 